\title{
Ergonomic assessment of enhanced protection under body armour combat shirt neck collars
}

\author{
John Breeze, ${ }^{1}$ C J Granger, ${ }^{2}$ T D Pearkes, ${ }^{3}$ J C Clasper ${ }^{1,4}$
}

\begin{abstract}
${ }^{1}$ Academic Department of Military Surgery and Trauma, Royal Centre for Defence Medicine, Birmingham Research Park, Birmingham, UK

23 Medical Regiment, Catterick, North Yorkshire, UK ${ }^{3} 19$ Regiment Royal Artillery, Tidworth, Wiltshire, UK ${ }^{4}$ The Royal British Legion Centre for Blast Injury Studies at Imperial College London, London, UK
\end{abstract}

\section{Correspondence to} Maj John Breeze, Academic Department of Military Surgery and Trauma, Royal Centre for Defence Medicine, Birmingham Research Park, Vincent Drive, Birmingham B15 2SQ, UK johno.breeze@gmail.com

Received 29 April 2013 Revised 22 May 2013 Accepted 1 June 2013 Published Online First 11 July 2013

To cite: Breeze J, Granger CJ, Pearkes TD, et al. J R Army Med Corps 2014;160:32-37.

\section{ABSTRACT}

Introduction Combat neck injury due to explosively propelled fragments is a significant cause of mortality and long-term morbidity in UK soldiers deployed on current operations. Reinforcing the collar of the existing under body armour combat shirt (UBACS) has been suggested as a potential method for reducing the incidence of combat neck injury.

Method 20 soldiers serving in Afghanistan objectively compared three designs of enhanced protection UBACS (EP-UBACS) using 10 representative military tasks against a baseline of a standard UBACS. Each EP-UBACS design was trialled using three constituent materials: two layers of para-aramid felt, one layer of ultra high molecule weight polyethylene (UHMWPE) felt or two layers of a silk fabric. Subjective assessment of these nine configurations in terms of comfort, heat dissipation and overall acceptability were compared with the standard UBACS using a $\chi^{2}$ test.

Results All military tasks could be performed with all nine configurations of EP-UBACS. Although silk was the most comfortable material, it was not functionally practical in any of the three designs. Crossover collars incorporating UHMWPE or para-aramid were the only two of the nine configurations to demonstrate similar user acceptability to a standard UBACS.

Conclusions The EP-UBACS has the potential to provide neck protection without reducing performance incorporating materials analogous to either of the felts assessed in this study. The collar should provide standoff from the skin to improve heat dissipation and comfort, which can be maximised by changing the current UBACS collar shape to one that crosses over at the front. Should a zip be desired, it should be moved to one side of the midline to reduce rubbing on the chin and be covered with ballistic protective material. Additional semi-circles of silk beneath the collar at the front and back would improve protection without affecting comfort.

\section{INTRODUCTION}

Combat neck injury due to explosively propelled fragments is a significant cause of mortality and long-term morbidity in UK service personnel deployed to Afghanistan. ${ }^{1}$ Currently, protection against such wounds is in the form of a detachable collar that attaches to the top of the current Mark IV OSPREY body armour. ${ }^{1-3}$ This body armour is routinely worn over an under body armour combat shirt (UBACS) developed for sweat and heat dissipation. The neck collar design has remained unaltered through all four iterations of OSPREY, despite the collars being disliked and rarely worn. ${ }^{3}$ Recent post-mortem analysis of 5 years of combat neck injuries (2006-2010) sustained by UK soldiers

\section{Key messages}

An enhanced protection under body armour combat shirt can potentially provide ballistic protection to the neck without reducing performance.

- The collar should provide stand-off from the skin to improve heat dissipation and comfort.

- The collar should use ballistic protective material analogous to one layer of the ultra high molecule weight polyethylene felt or two layers of the para-aramid felt assessed in this study.

- The current zip should be moved to one side of the midline to reduce rubbing on the chin.

- A zip would require covering with ballistic protective material to maximise potential protection.

- Additional semi-circles of silk beneath the collar at the front and rear would improve protection without affecting comfort.

demonstrated that these collars could potentially have mitigated many injuries from explosively propelled fragments had they been worn; however, no soldier during this period was wearing his or her collar at the time of neck injury. ${ }^{1}$

This finding led to the first published ergonomic assessment of neck protection and demonstrated that the OSPREY neck collar was the worst performing design when compared with the collars of five other nations. ${ }^{2}$ The design prevented a soldier from firing in the prone position when it was worn, which is an essential dismounted task in the current operational environment and there was therefore a considerable drive at the Ministry of Defence to develop a better method of protecting the neck from explosively propelled fragments. A dedicated neck protection programme was established in 2010 as a collaboration among the Royal Centre for Defence Medicine, Dstl Porton Down and Defence Equipment and Support, the primary focus of which has been on refining the design of the OSPREY neck collar. ${ }^{3}$

Part of the difficulty in making objective comparisons between designs has been a lack of a standardised framework of tests and measurements. ${ }^{4-10}$ Our first trial used military representative military tasks to compare between prototypes; ${ }^{2}$ one of the tasks (vehicle ingress) had been described in the only openly available UK publication at that time and the remainder were generated by a military judgement panel to be representative of what a UK 
soldier would be expected to perform on operations such as firing a rifle in the prone position or a casualty drag. Specific design features such as crossing over of the collar to enable firing in the prone position were identified and used in future prototypes. ${ }^{2}$ However, these military representative tasks still remained somewhat subjective and therefore in the second trial of neck collars physiological measurements such as heart rate and skin temperature were used to attempt to differentiate between tasks more objectively. ${ }^{3}$ Although such measurements had enabled comparisons between body armour vests previously, ${ }^{5}$ the differences were too small to be detected when just the neck collars alone were compared. ${ }^{3}$

An alternative and novel method of providing protection to the neck has recently been identified ${ }^{3}$ which involves incorporating ballistic protective material into the collar of the UBACS, a concept termed the enhanced protection UBACS (EP-UBACS). This concept could potentially act as an irreducible minimum amount of protection (Tier 1 level protection) with the option of wearing an OSPREY neck collar in addition (Tier 2 level protection) during situations of increased threat; such a tiering system is currently being used successfully for pelvic protection in the deployed UK military. ${ }^{11}$ The EP-UBACS concept was briefly assessed as part of a larger trial of OSPREY ballistic neck collars $^{3}$ using thick ballistic felt in the collar of the current UBACS shirt. Although it was liked in principle, the ballistic protective material used was perceived as being too thick when the collar was zipped up. It did however demonstrate potential and further ergonomic assessment was recommended. Subsequent surface wound mapping (SWM), the process by which the entry wound locations of penetrating wounds are recorded graphically, ${ }^{1}$ has also demonstrated obvious areas lacking in ballistic protection (Figure 1). This was most noticeable at the front and rear between the bottom of the EP-UBACS collar and the top of the OSPREY vest. One goal of our research programme is now to develop an EP-UBACS design that would provide ballistic protection to all parts of the neck and chest not covered by the OSPREY vest but which would have no significant effect on soldier acceptability and military performance.
The aim of this assessment was therefore to compare combinations of ballistic protective materials in three novel EP-UBACS collar designs with the existing UBACS when worn in a representative operational environment.

\section{METHOD}

An ergonomics assessment was undertaken in Afghanistan on Op HERRICK 17A in October 2012. The ambient temperature and humidity ranged between $35^{\circ} \mathrm{C}$ and $41^{\circ} \mathrm{C}$ and $19 \%$ and $31 \%$. In all, 20 deployed UK servicemen (10 infantry soldiers, five Royal Logistic Corps personnel and five combat medical technicians) ranging in rank from private soldier to sergeant assessed each prototype. Participants were chosen to represent the broad range of UK service personnel who would be expected to wear these garments on a daily basis.

Three designs were assessed (EP-UBACS 1, EP-UBACS 2 and EP-UBACS 3), each with one of three different constituent ballistic protective materials (Figure 2); each of the nine EP-UBACS configurations were compared with each other and with a standard unmodified UBACS. The EP-UBACS 1 design (Figure 1A) was the most similar to the existing UBACS, with the only modification being the incorporation of ballistic protective material into the collar (Figure 1D), and was analogous to that tested in the previous trial. ${ }^{3}$ The EP-UBACS 2 prototype was developed using a design identical to the EP-UBACS 1, but with an additional semicircle of ballistic protective material at the front and rear (Figure 1B) which could be seen on SWM to cover those areas of the upper thorax not currently covered by the OSPREY vest (Figure 1E). The last of the three prototypes, EP-UBACS 3, was a standard UBACS shirt with the collar modified to crossover at the front (Figure 1C); such a design was developed to enable the collar to stand up without the requirement of a zip (Figure 1F).

The following three combinations of ballistic protective materials were used with values for mass per unit area (areal density) and thickness derived from values supplied by the manufacturers: two layers of a para-aramid felt (mass per unit area $255 \mathrm{~g} / \mathrm{m}^{2}$ per layer), one layer of ultra high molecular weight
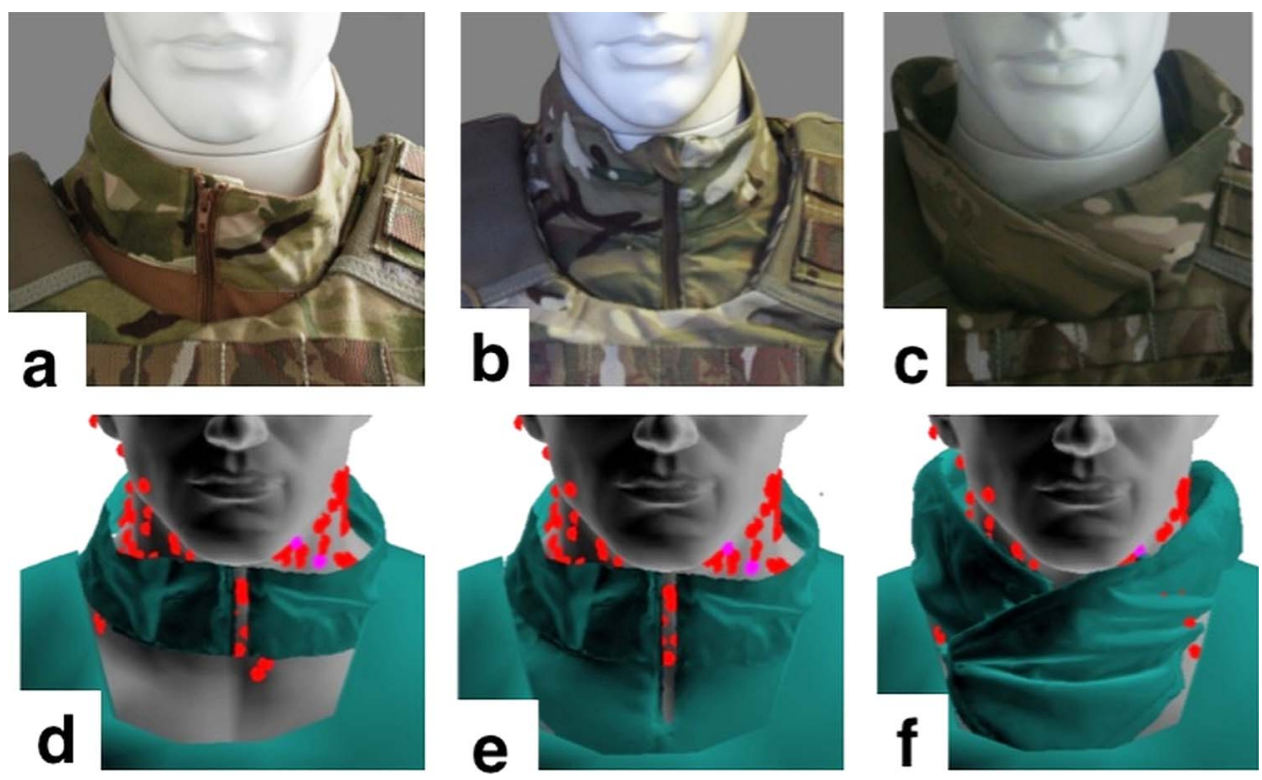

Figure 1 Enhanced protection under body armour combat shirt (EP-UBACS) 1-3 designs fitted on an anatomical manikin (A-C) with pictorial comparisons of their potential ballistic protective qualities using surface wound mapping (D-F); for security reasons, the wound entry locations in this case are imaginary. 


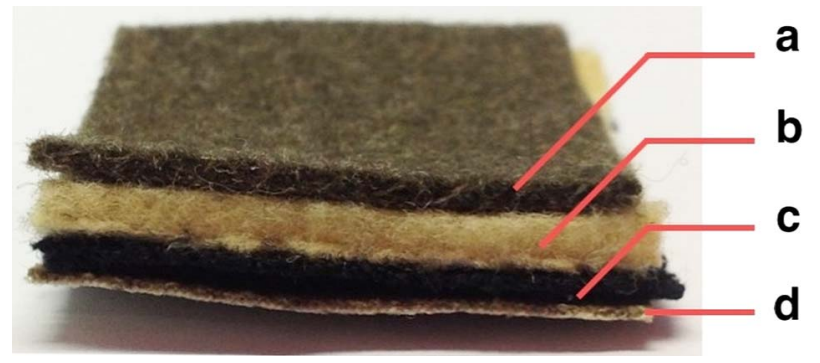

Figure 2 Ballistic protective and cover materials used; (A) Paraaramid felt (one layer); (B) Ultra high molecular weight polyethylene felt (one layer); (C) Silk fabric (two layers); and (D) Cover material.

polyethylene (UHMWPE) felt (mass per unit area $600 \mathrm{~g} / \mathrm{m}^{2}$ ) or two layers of a silk fabric (mass per unit area $150 \mathrm{~g} / \mathrm{m}^{2}$ per layer). Two layers of silk fabric were $0.9 \mathrm{~mm}$ in depth in total, a single layer of para-aramid felt was $1.0 \mathrm{~mm}$, and a single layer of UHMWPE felt was $2.1 \mathrm{~mm}$. Two layers of silk fabric were chosen as this material has been successfully used in Tier 1 pelvic protection ${ }^{11}$ and has demonstrated a reduction in the incidence of pelvic injuries from explosive fragments. Two layers of para-aramid felt were chosen as such a combination has recently been suggested as the most effective method of protecting the extremities from explosive fragmentation without unduly increasing heat and weight. ${ }^{12}$ One layer of UHMWPE was chosen as this was half the ballistic protective material (in terms of thickness and mass per unit area) than that in the previously assessed EP-UBACS collar, which was subjectively felt to be too thick. ${ }^{3}$ Due to logistical difficulties getting UHMWPE from the UK, this material only became available to us at the end of the assessment and had to be taken from the EP-UBACS 3 designs. We were therefore only able to assess 15 participants with this material in the EP-UBACS 1 design and 10 participants in the EP-UBACS 2 design (as this design required more of the material).

The ballistic protective materials were enclosed by a lightweight knitted fabric front and rear cover material (mass per unit area $80 \mathrm{~g} / \mathrm{m}^{2}$ ) that was identical in all configurations. Each participant assessed the standard UBACS first, followed by the

\begin{tabular}{ll} 
Table 1 List of representative dismounted close combat (DCC) \\
and mounted close combat (MCC) tasks undertaken in this \\
assessment \\
\hline Type & Task \\
\hline DCC & Put on body armour with OSPREY neck collars attached \\
DCC & Fire weapon prone \\
DCC & Fire weapon kneeling \\
DCC & Fire weapon standing \\
DCC & Leopard crawl \\
DCC & Route clearance with VALLON and buried explosive device confirmation \\
& drill \\
DCC & Put on and take off standard issue G10 respirator \\
MCC & Ingress through rear door of Mastiff, sit down and fasten seat-belt \\
MCC & Ingress through turret of Mastiff into 'Top Cover' position \\
MCC & Fire general purpose machine gun from top cover position in Mastiff \\
DCC & Take off body armour with OSPREY neck collars attached \\
\hline
\end{tabular}

nine EP-UBACS prototype configurations in a random order, produced by a random number generator. Participants performed each task once and tasks took between 2 and 4 min each to perform. Participants were unaware of which ballistic protective material was in each collar. It was also possible to anonymise between the EP-UBACS 1 and 2 designs by adding a single semicircle of thin non-ballistic protective material at the front and rear of the standard UBACS and each EP-UBACS 1 prototype to mimic the appearance of the EP-UBACS 2.

\section{Objective assessments}

A range of static and dynamic representative military tasks (Figure 3, Table 1) chosen from the limited available evidence $^{4-10}$ in conjunction with expert opinion following our two previous ergonomic assessments of neck protection ${ }^{2} 3$ were used to provide objective assessment between configurations. All tasks were undertaken using standardised clothing and equipment, including the issued $35 \mathrm{~L}$ rucksack, a Mark 7 helmet and the current short OSPREY neck collars attached to the ballistic vest. Each rucksack was filled with bags of saline to give an additional mass of $10 \mathrm{~kg}$. All participants were asked after each task

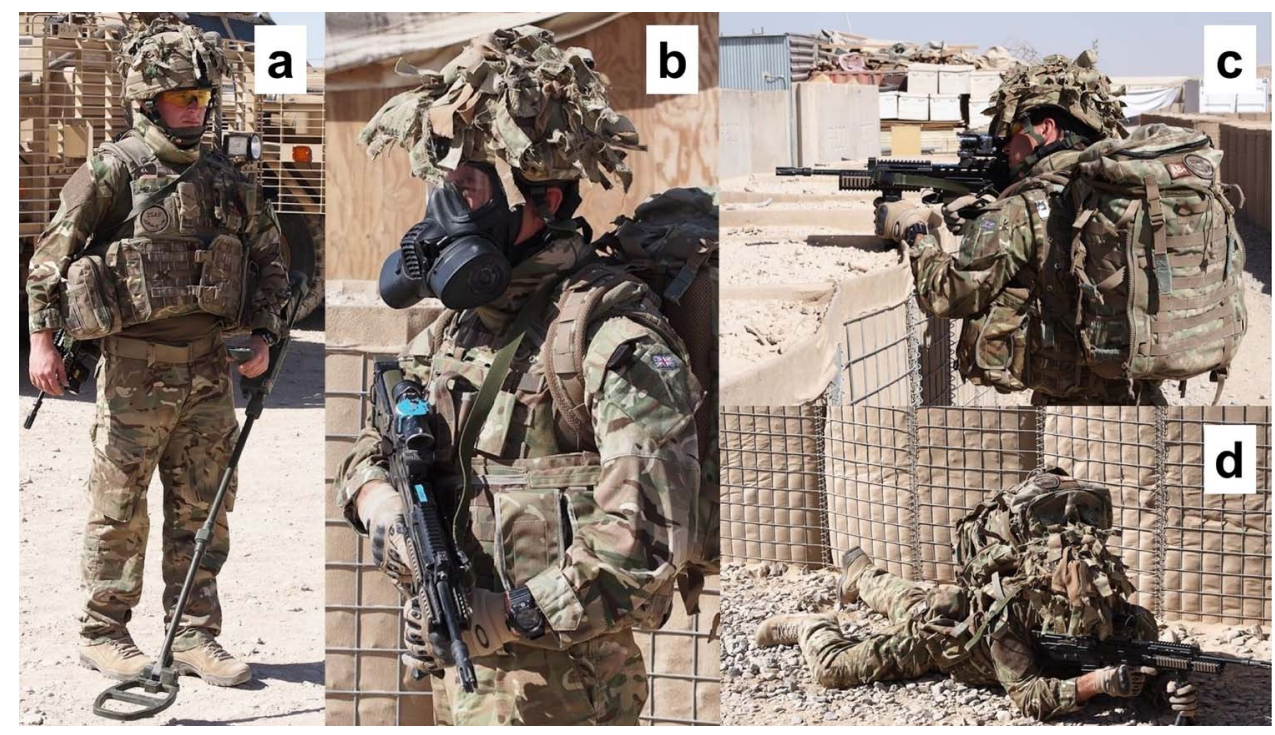

Figure 3 Examples of representative military tasks: (A) VALLON route clearance, (B) Use of G10 respirator, (C) Firing standing and (D) Firing prone. 
Table 2 Objective assessments of prototypes using representative military tasks outlined in Table 1

\begin{tabular}{|c|c|c|c|c|c|c|c|c|c|c|c|}
\hline \multirow[b]{2}{*}{ Configuration } & \multicolumn{11}{|c|}{ Task } \\
\hline & A & B & C & D & E & $F$ & G & H & I & J & K \\
\hline \multicolumn{12}{|l|}{ Standard UBACS } \\
\hline \multicolumn{12}{|c|}{ EP-UBACS 1 (two layers silk) } \\
\hline \multicolumn{12}{|c|}{ EP-UBACS 1 (two layers para-aramid) } \\
\hline \multicolumn{12}{|c|}{ EP-UBACS 1 (one layer UHMWPE) } \\
\hline \multicolumn{12}{|c|}{ EP-UBACS 2 (two layers silk) } \\
\hline \multicolumn{12}{|c|}{ EP-UBACS 2 (two layers para-aramid) } \\
\hline \multicolumn{12}{|c|}{ EP-UBACS 2 (one layer UHMWPE) } \\
\hline \multicolumn{12}{|c|}{ EP-UBACS 3 (two layers silk) } \\
\hline \multicolumn{12}{|c|}{ EP-UBACS 3 (two layers para-aramid) } \\
\hline EP-UBACS 3 (one & & & & & & & & & & & \\
\hline
\end{tabular}

whether they could complete the task without constraints, complete the task but with certain constraints or whether they were unable to complete the task. Examples of constraints included having more difficulty to sight an aimed shot or more effort required to extend the neck in the prone position. It required more than $90 \%$ of participants to be able to complete a given task without constraints for a configuration to be given a white box in Table 2. This cut-off was derived from an expert military judgement panel and had been used in a previous ergonomics assessment. ${ }^{3}$ If more than $10 \%$ of participants could only complete a task with constraints, the box was shaded grey.

\section{Subjective assessment}

The effect of each configuration on perceived comfort, equipment integration, heat dissipation and overall acceptability was recorded using a 5-point Likert scale. This assessment method has previously been used successfully in determining the impact of body armour on lower body movement. ${ }^{10}$ An anonymous questionnaire was given to each participant after all tasks with each configuration were completed. A non-commissioned officer independent of our team collected the completed questionnaires and compiled the results. The overall acceptability scores from the Likert scales were converted to binomial data by combining all agree and disagree responses into two categories of 'acceptable' and 'unacceptable', enabling a $\chi^{2}$ test to be performed. The null hypothesis was that there was no difference in acceptability between that configuration and the standard UBACS. Statistical analysis was undertaken using the IBM SPSS statistical package (V.20.0.1), with statistical significance defined as a $\mathrm{p}<0.05$.

\section{RESULTS}

The height and weight of participants ranged between 175 and $193 \mathrm{~cm}$ (mean $186 \mathrm{~cm}$ ) and 71 and $88 \mathrm{~kg}$ (mean $78 \mathrm{~kg}$ ). Table 2 demonstrates the objective results using representative military tasks. It can be clearly seen that all tasks could be performed with all configurations using the threshold of $90 \%$. Configurations using UHMWPE were most likely to result in tasks that could only be performed with reduced performancethe prone firing and leopard crawl tasks with UHMWPE caused the greatest difficulty and resulted in 9/15 (60\%) participants being unable to complete the task with the EP-UBACS 1 and 5/ 10 (50\%) with the EP-UBACS 2 configurations, respectively. Participants noted that the UHMWPE collar in these configurations was stiff and hit the underneath of the chin, limiting their ability to assume the prone position. The collar again rubbed on the chin when trying to make an aimed shot with two layers of para-aramid in the EP-UBACS 1 and 2 designs when firing prone. Adding the EP-UBACS 1 and 2 results together, this resulted in 7/25 (28\%) using UHMWPE collars and $6 / 40(15 \%)$ of participants using para-aramid collars only being able to complete these tasks with constraints.

The subjective participant assessment for each configuration is given in Table 3. The EP-UBACS 3 designs incorporating either UHMWPE or two layers of para-aramid felt were the only configurations to demonstrate no significant difference in user acceptability compared with a standard UBACS $(\mathrm{p}=0.57$ and 0.89 , respectively). Heat dissipation was the main reason for a configuration being unacceptable and was primarily found in the EP-UBACS 1 and 2 designs. Heat dissipation was worse with UHMWPE in these collars than two layers of para-aramid with only $2 / 20(10 \%)$ participants finding the former material acceptable compared with $7 / 20$ (35\%) for the latter. UHMWPE in the collar of both the EP-UBACS 1 and 2 collars also caused so much discomfort due to it rubbing on the undersurface of the chin that $15 / 20(75 \%)$ defined it as unacceptable with each design. Reinforcing collars with silk provided no statistical difference in perceived heat dissipation for the EP-UBACS 1, 2 and 3 designs compared with a standard UBACS ( $p$ values of 0.094 , 0.062 and 0.13 , respectively). All 20 participants found that the silk was the most comfortable of the three materials when lying directly next to the skin, but the silk collars would not stand upright in any of the three designs after repeated use, which was most evident with the EP-UBACS 3 design (Figure 4A).

\section{DISCUSSION}

The aim of this assessment was to compare three novel EP-UBACS collar designs using different combinations of ballistic protective materials with the existing UBACS. The EP-UBACS 1 design was the most similar to the existing UBACS, with the only modification being the incorporation of ballistic protective material into the collar. Although all military tasks could be performed with all three materials in the EP-UBACS 1 design, the UHMWPE was unacceptable in terms of comfort and heat dissipation, as previously reported ${ }^{3}$ when a material was used that was analogous in thickness and flexibility to two layers of the UHMWPE felt used in our current study, suggesting that UHMWPE should not be used if an EP-UBACS 1 
Table 3 Subjective assessments of prototypes ranked using 5-point Likert scale

\begin{tabular}{lllll}
\hline Configuration & Comfort & Equipment integration & Heat dissipation & Overall acceptability \\
\hline Standard UBACS & 1 & 1 & 2 & 2 \\
EP-UBACS 1 (two layers silk) & 1 & 1 & 2 & 3 \\
EP-UBACS 1 (two layers para-aramid) & 2 & 1 & 5 & 3 \\
EP-UBACS 1 (one layer UHMWPE) & 5 & 1 & 5 & 4 \\
EP-UBACS 2 (two layers silk) & 2 & 1 & 5 & 3 \\
EP-UBACS 2 (two layers para-aramid) & 3 & 1 & 5 & 3 \\
EP-UBACS 2 (one layer UHMWPE) & 5 & 1 & 1 & 5 \\
EP-UBACS 3 (two layers silk) & 2 & 1 & 2 & 4 \\
EP-UBACS 3 (two layers para-aramid) & 1 & 3 & 1 & 2 \\
EP-UBACS 3 (one layer UHMWPE) & 2 & 2 & 1 \\
\hline 1=strongly agree, 2=agree, 3=neutral, 4=disagree, 5=strongly disagree. & \\
EP-UBACS, enhanced protection under body armour combat shirt; UBACS, under body armour combat shirt; UHMWPE, ultra high molecule weight polyethylene.
\end{tabular}

concept was chosen. Silk fabric was acceptable both in terms of military task performance and subjective soldier acceptability and is currently being used successfully for Tier 1 pelvic protection. ${ }^{11}$ However, even with the collar zipped up, the silk caused the collar to fall down after repeated use which would result in an unacceptable reduction in coverage of the neck, thereby negating the effect of the ballistic protective material. The current zip caused rubbing on the undersurface of the chin when zipped up and in future iterations of this design the zip should be relocated to one side of the midline and should stop just above the upper limit of the OSPREY vest. SWM has demonstrated that any such zip should be covered by a layer of ballistic material to ensure there are no gaps in potential protection. Should an EP-UBACS 1 design be chosen then a felt material analogous in
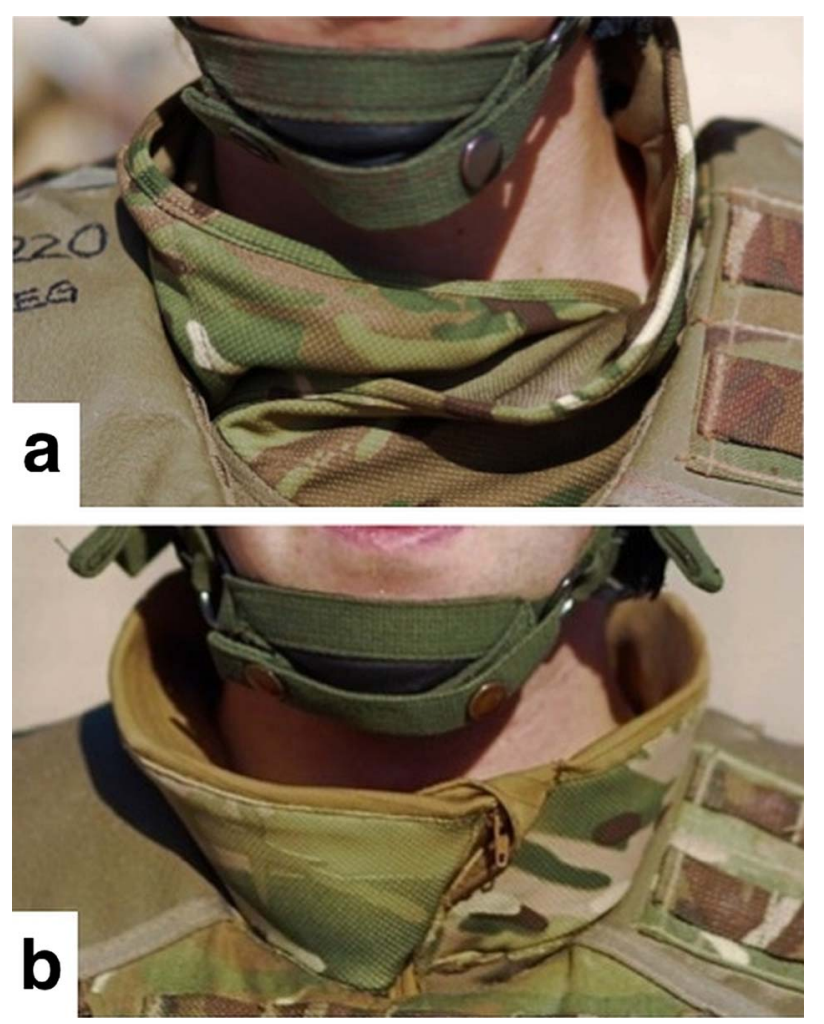

Figure 4 Silk alone in the collar causes it to drop down (A); suggested design features for future prototypes including standoff from skin and zip to one side of the midline (B). terms of material properties to the para-aramid in this study should be used.

The EP-UBACS 2 design was developed following SWM analysis demonstrating that reinforcing the collar of the existing UBACS alone (the EP-UBACS 1 design) would potentially result in an area between the top of the OSPREY vest and the reinforced neck collar that lacked protection. Configurations that used additional semicircles of UHMWPE or para-aramid at the front and rear demonstrated identical task performance as EP-UBACS 1 but worse subjective soldier acceptability. This was particularly found with regard to comfort and heat dissipation with participants stating that these materials stuck to their chest and caused them to sweat more. The addition of a semicircle of silk made no subjective difference to soldier acceptability compared with an unmodified UBACS, confirming work done on the comfort of silk next to the skin for Tier 1 pelvic protection. ${ }^{11}$ These findings would suggest that although UHMWPE and para-aramid felts are not recommended for these additional semicircles of ballistic protective materials, two layers of a silk fabric would be a potential solution even if this was not used in the collar itself.

In the EP-UBACS 3 design the standard UBACS collar was altered to one that crosses over at the front, enabling the zip to be removed while maintaining skin coverage. This design had greater stand-off from the skin than the standard UBACS collar of the EP-UBACS 1 and 2, the effect of which appeared dependent on the material used in the collar. The silk collar collapsed from the first use negating its protective function and should not be used in such a design. The UHMWPE and para-aramid collars in the EP-UBACS 3 demonstrated no significant difference in subjective user acceptability from a standard UBACS. However, this stand-off resulted in interference with the OSPREY neck collar preventing participants from assuming the prone position required to fire the rifle or perform a leopard crawl. These difficulties could potentially be negated by reducing the stand-off of the collar from the skin but still providing enough of a gap to enable air movement. Should a zip still be desired with a crossover collar design, then the zip need only go up to the point where the collar layers cross.

There are potential limitations within this trial, primarily the inability to blind the EP-UBACS 3 configurations due to its very different design raising the possibility that participants might modify an aspect of their behaviour being experimentally measured simply in response to the fact that they know they are being studied. ${ }^{13}$ It is noted that all 20 participants did not assess the EP-UBACS 1 and 2 configurations that used UHMWPE felt; 
however, even the slightly smaller participant samples clearly demonstrated that neither of these configurations would be acceptable in their current form. Although a period of 2 weeks is insufficient to confidently predict whether these prototypes will be accepted in the long term, we believe that our results provide sufficient evidence to justify the financial impact of procuring a far larger number of the successful EP-UBACS 2 and 3 designs. We recommend that all UK soldiers deploying to Afghanistan from now be issued with the EP-UBACS 1 design instead of their existing UBACS as this would be the easiest to modify; consideration should then be made to replacing this with either the EP-UBACS 2 or 3 designs.

\section{CONCLUSIONS}

This trial has demonstrated that some configurations of EP-UBACS would potentially provide ballistic protection to the neck without adversely affecting soldier performance and further prototypes should be made using a combination of the best design features identified in this study (Figure 4B). The neck collar should provide stand-off from the skin, to improve heat dissipation and overall comfort and should incorporate a ballistic protective material, or layers of material, that provide both mass per unit area and thickness akin to one layer of UHMWPE felt or two layers of para-aramid as assessed in this study. Consideration should be made to adding a semi-circle of silk fabric at the front and back. Should a zip still be desired, it should be relocated to one side of the midline to reduce rubbing on the chin but should stop just above the upper limit of the OSPREY vest. Should a crossover collar design be chosen, then the zip need only go up to the point where the collar layers cross. SWM however has demonstrated that any such zip should be covered by a layer of ballistic material to ensure there are no gaps in potential protection. Although further refinement of the designs is recommended, this should not delay the implementation of the EP-UBACS into service, which has the potential to reduce the mortality and morbidity experienced by UK service personnel in Afghanistan.

Acknowledgements The authors would like to thank Adrian Randall at Defence Equipment and Support as well as Robert Robinson Collins at the Defence Science and Technology Laboratory, Porton Down, for their invaluable support in arranging for the varying iterations of these prototypes to be made and sent out to Afghanistan, often at very short notice.

Contributors Planning: JB; Conduct: JB, CG and TP; Reporting: JB and JC. Competing interests None.

Ethics approval Ethical approval was confirmed as this study was agreed to constitute an assessment for which a generic protocol had already been approved through the Ministry of Defence Research Ethics Committee process.

Provenance and peer review Not commissioned; externally peer reviewed.

\section{REFERENCES}

1 Breeze J, Allanson-Bailey LS, Hunt NC, et al. Mortality and morbidity from UK combat neck injury. J Trauma 2012;72:969-74.

2 Breeze J, Watson $\mathrm{CH}$, Horsfall I, et al. Comparing the human factors of neck collars in different military body armour systems. Mil Med 2011;176:1274-7.

3 Breeze J, Clasper JC. Ergonomic assessment of future methods of ballistic neck protection. Mil Med 2013. In press.

4 Horsfall I, Champion SM, Watson CH. The development of a quantitative flexibility test for body armour and comparison with wearer trials. Appl Ergon 2005;36:283-92.

5 Ricciardi R, Deuster PA, Talbot LA. Metabolic demands of body armor on physical performance in simulated conditions. Mil Med 2008;173:817-24.

6 Harman EA, Gutekunst DJ, Frykman PN, et al. Prediction of simulated battlefield physical performance from field-expedient tests. Mil Med 2008;173:36-41.

7 Bogdan A, Marszałek A, Majchrzycka K, et al. Aspects of applying ergonomic tests in the evaluation of ballistic body armours using the example of ballistic vests. $J$ Textile Sci Eng 2012;2:123.

8 Caldwell JN, Engelen L, van der Henst C, et al. The interaction of body armor, low-intensity exercise, and hot-humid conditions on physiological strain and cognitive function. Mil Med 2011;176:488-93.

9 Payne W, Harvey J. A framework for the design and development of physical employment tests and standards. Ergonomics 2010;53:858-71.

10 Park H. Impact of Body Armour and Load Carriage on Lower Body Movement. Release date: 18 October 2012. Proquest dissertation publishing. ISBN: 978-1249873426

11 Lewis EA, Pigott MA, Randall A, et al. The development and introduction of ballistic protection of the external genitalia and perineum. J R Army Med Corps 2013;159 (Suppl):i15-7.

12 Sakaguchi S, Carr D, Horsfall I, et al. Protecting the extremities of military personnel: fragment protective performance of one- and two-layer ensembles. Textile Res J 2012;82:1295-303.

13 McCarney R, Warner J, lliffe S, et al. The Hawthorne Effect: a randomised, controlled trial. BMC Med Res Methodol 2007;7:30. 